\title{
Building 3D polymer and metal microstructures
}

\author{
Nobuyuki Takeyasu, Florian Formanek, Takuo Tanaka, \\ Atsushi Ishikawa, Kenta Chiyoda, and Satoshi Kawata
}

A combination of metal-attractive and metal-resistant polymers enables selective deposition of metal with submicron features onto a $3 D$ template structure.

Microstructures with nanosized features are of great interest in various research fields because of their unique properties. But fabrication techniques are still being developed. Electron-beam and focused-ion-beam lithography are frequently used for 2D patterning on these scales, but are not well suited for fabrication of 3D structures. Researchers are also still finding ways to construct devices using materials with specific properties and functions. We describe work that focuses electrically conductive metal and nonconductive polymers as the building materials. Our method can fabricate 3D metal/polymer microstructures intended for electrical and optical applications.

Weused two-photonabsorption(TPA)inUV-cured resin tomake $3 \mathrm{D}$ structures. When a high-power laser $(\lambda=800 \mathrm{~nm})$ is focused into the resin, a tiny area at the focus $(\sim 100 \mathrm{~nm})$ is polymerized by the TPA process. This method can be used to form 3D structures by scanning the focusing spot in the resin. ${ }^{1-3}$ Production efficiency can be enhanced by using a microlens array to enable multiple parallel processing. ${ }^{4}$ Several groups have also reported using TPA for direct metal reduction, to make both $2 \mathrm{D}$ and 3D structures. ${ }^{5-7}$

After fabricating 3D polymer templates, we used electrochemical metal deposition to coat the structure. ${ }^{8,9}$ The success of this method, however, depends strongly on the chemical properties of the polymer surface. Because TPA offers the advantage of localized polymerization within the focusing spot, one can use it to fabricate a 3D structure composed of more than one polymer. For example, part of a structure could be made from a material that accepts metal deposition readily, while other parts can be made from metal-resistant polymers. This allows us to build a partially metal coated 3D polymer structure.

We used metal-resistant and metal-attractive UV-curable resins to fabricate another $3 \mathrm{D}$ polymer template. The commercially available resin KC1102 (from JSR) was used as a

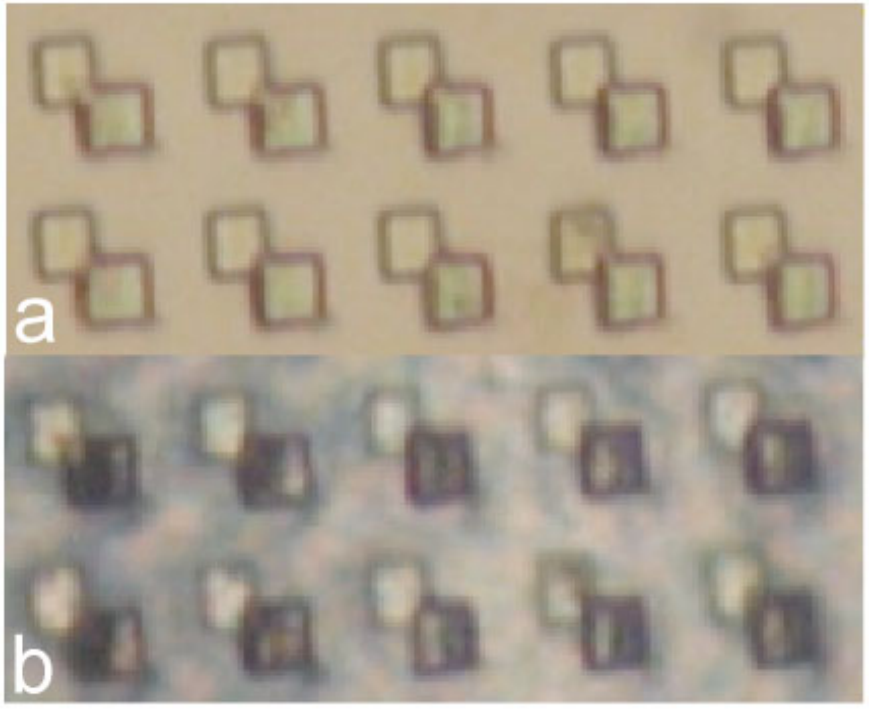

Figure 1. Shown are microscope images of pairs of polymer sheets made by TPA microfabrication. The polymer that resists metallization is on the upper left of each pair, while the polymer that accepts metallization is on the lower right. Each square sheet measures $2 \times 2 \mu m$. (a) The array was soaked in a silver solution for 6h. (b) The array was electroless plated.

metal-resistant resin. The metal-attractive resins were prepared using either a methacrylamide (MAA, from Aldrich) or a (9H-carbazol-9-yl) ethyl methacrylate (CEM, also from Aldrich). We mixed either MAA with KC1102 or CEM with another resin (Z7012C from JSR). We used a microlens array to form multiple beams from one femtosecond Ti:sapphire laser beam. ${ }^{8}$ When the beams were focused into the resin with an objective lens and scanned, they wrote polymerized 3D templates with submicron features. The templates were immersed in silver-plating solution, and then we observed the results with a scanning electron microscope (SEM) and an optical microscope.

In our first experiment, we prepared pairs of polymer sheets with KC1102 and KC1102+MAA and confirmed that silver is

Continued on next page 
a

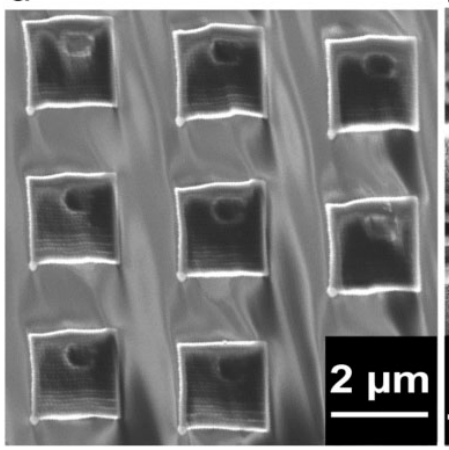

b

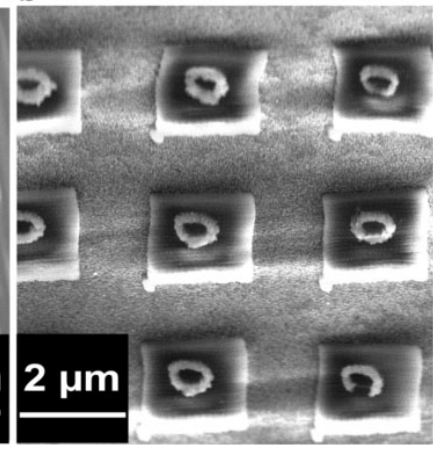

Figure 2. Scanning electron microscope (SEM) images of polymer templates show site-selective silver deposition. (a) Squares are formed from KC1102 polymer, while the rings are made of KC1102+MAA. (b) After electrochemical deposition, the ring structures are coated with silver, but the squares are not.

selectively deposited on the attractive polymer (see Figure 1). Amide groups in the MAA attracted the silver. In another experiment, we fabricated layered structures, as shown in Figure 2 . The template consists of $1 \mu \mathrm{m}$-diameter metal-attractive polymer rings on a $2 \times 2 \mu \mathrm{m}$ sheet of metal-resistant polymer. After deposition, only the ring structures were covered with silver.

The template made using CEM is shown in Figure 3. The polymer template was prepared from CEM-Z7012C and KC1102. Silver was selectively deposited on the former. When the polymer is exposed to UV light, the carbazole group in the CEM emits electrons, which then reduce the silver ions.

\section{Conclusion}

To make 3D metal-and-polymer structures on the nano- and micrometer scale, we proposed and demonstrated a method that combines TPA microfabrication and electrochemical metal deposition. Both the resins we modified attract metal, although the commercially available resin resists metal deposition. The localization of polymerization through TPA allows us to make $3 \mathrm{D}$ polymeric templates consisting of parts that attract or resist metallization. This in turn lets us build 3D microstructures from these materials. This technique can be used to align metal structures supported by polymer structures.

\section{Author Information}

\section{Nobuyuki Takeyasu, Florian Formanek, and Takuo Tanaka}

Nanophotonics Laboratory

RIKEN (The Institute of Physical and Chemical Research)

Wako, Japan a

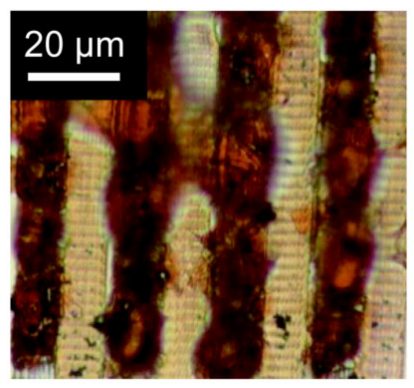

b

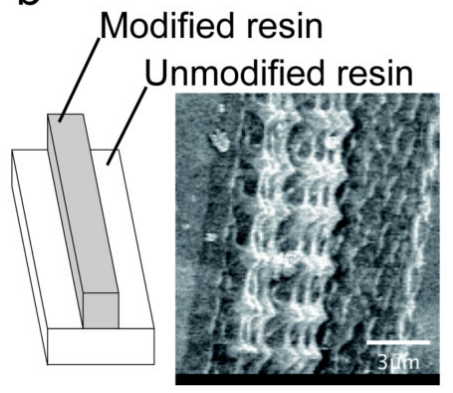

Figure 3. (a) The transmission image shows silver deposited on polymer lines using UV irradiation. (b) The SEM image is of a silver/polymer layered structure.

\section{Atsushi Ishikawa and Satoshi Kawata \\ Nanophotonics Laboratory \\ RIKEN (The Institute of Physical and Chemical Research) \\ Wako, Japan}

Department of Applied Physics

Osaka University

Suita, Japan

\section{Kenta Chiyoda}

Nanophotonics Laboratory

RIKEN (The Institute of Physical and Chemical Research)

Wako, Japan

Department of Physics

Gakushuin University

Toshima-ku, Japan

\section{References}

1. B. H. Cumpston, S. P. Ananthavel, S. Barlow, D. L. Dyer, J. E. Ehrlich, L. L. Erskine, A. A. Heikal, S. M. Kuebler, S. Lee, D. McCord-Maughon, J. Qin, H. Röckel, M. Rumi, X.-L. Wu, S. R. Marder, and J. W. Perry, Two-photon polymerization initiators for three-dimensional optical data storage and microfabrication, Nature 398 (6722), pp. 51-54, 1999.

2. S. Kawata, H.-B. Sun, T. Tanaka, and K. Takada, Finer features for functional microdevices, Nature 412 (6848), pp. 697-698, 2001.

3. K. K. Seet, V. Mizeikis, S. Matsuo, S. Juodkazis, and H. Misawa, Three-dimensional spiral-architecture photonic crystals obtained by direct laser writing, Adv. Mater. 17 (5), pp. 541-545, 2005.

4. J. Kato, N. Takeyasu, Y. Adachi, H.-B. Sun, and S. Kawata, Multiple-spot parallel processing for laser micronanofabrication, Appl. Phys. Lett. 86 (4), p. 044102, 2005.

5. F. Stellacci, C. A. Bauer, T. Meyer-Friedrichsen, W. Wenseleers, V. Alain, S. M. Kuebler, S. J. K. Pond, Y. Zhang, S. R. Marder, and J. W. Perry, Laser and electronbeam induced growth of nanoparticles for 2D and 3D metal patterning, Adv. Mater. 14 (3), pp. 194-198, 2002.

6. T. Baldacchini, A.-C. Pons, J. Pons, C. N. LaFratta, J. T. Fourkas, Y. Sun, and M. J. Naughton, Multiphoton laser direct writing of two-dimensional silver structures, Opt. Express 13 (4), pp. 1275-1280, 2005. 
7. T. Tanaka, A. Ishikawa, and S. Kawata, Two-photon-induced reduction of metal ions for fabricating three-dimensional electrically conductive metallic microstructure, Appl. Phys. Lett. 88 (8), p. 081107, 2006.

8. F. Formanek, N. Takeyasu, T. Tanaka, K. Chiyoda, A. Ishikawa, and S. Kawata, Three-dimensional fabrication of metallic nanostructures over large areas by two-photon polymerization, Opt. Express 14 (2), pp. 800-809, 2006.

9. F. Formanek, N. Takeyasu, K. Chiyoda, T. Tanaka, A. Ishikawa, and S.

Kawata, Selective electroless plating to fabricate complex three-dimensional metallic micro/nanostructures, Appl. Phys. Lett. 88 (8), p. 083110, 2006. 